\title{
Scar Ectopic Pregnancy
}

\author{
Madhuri Arvind Patel ${ }^{1}$
}

Received: 10 September 2015/Accepted: 29 October 2015/Published online: 21 November 2015

(C) Federation of Obstetric \& Gynecological Societies of India 2015

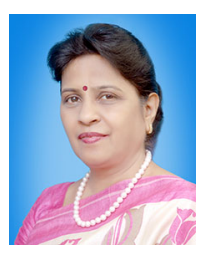

\begin{abstract}
About the Author
Dr. Madhuri Arvind Patel is currently working as Honorary Clinical Associate in OBGYN at Nowrosjee Wadia Maternity Hospital, Mumbai. At present she is Treasurer of FOGSI and First Assistant Editor of Journal of OBGYN of India. She is ExProfessor and HOD, ESIC-PGIMSR, MGMH, Mumbai, and Ex-Associate Professor from Grant Medical College and Sir JJ Group of Hospitals, Mumbai. She has over 22 years of teaching experience. She has undergone training on Clinical Research Methodology at IRRR and has been a Master Trainer for HIV in Pregnancy in Maharashtra. She was Joint Secretary FOGSI 2009 and was Chairperson of Study on Female Breast Committee FOGSI from 2010 to 2013.
\end{abstract}

\begin{abstract}
Scar ectopic pregnancy is the rarest form of ectopic pregnancy and has been increasingly diagnosed all over the world. This is a life-threatening form of abnormal implantation of embryo within the myometrium and fibrous tissues in a previous scar on the uterus, especially following caesarean section. With the increasing rate of caesarean section, there is a substantial increase in this condition with better understanding of this disease. The early and accurate diagnosis with timely management can prevent pregnancy complications such as haemorrhage, uterine rupture and can preserve fertility.
\end{abstract}

Madhuri Arvind Patel

drmadhuripatel@gmail.com

1 Nowrosjee Wadia Maternity Hospital, Mumbai, India
Keywords Scar ectopic pregnancy .

Abnormal implantation - Previous scar - Haemorrhage . Uterine rupture $\cdot$ Fertility

\section{Introduction}

Scar ectopic pregnancy is becoming increasing common all over the globe. It is novel and life-threatening form of abnormal implantation of embryo within the myometrium and the fibrous tissue of the previous scar following caesarean section; hysterotomy; dilation and curettage; abnormal placentation; surgery on uterus like myomectomy, metroplasty, hysteroscopy and manual removal of placenta.

Not much information is available on the natural history of this condition. However, the scar ectopic pregnancy is found to be the most common following caesarean section. In 1978, the first case of a caesarean scar (CS) ectopic 
pregnancy was reported in English medical literature [1]. With the increasing rate of caesarean section, there is a substantial increase in this condition with better understanding of this disease. The incidence of CS ectopic pregnancy varies from 1:1800 to 2216 pregnancies with rate of $0.15 \%$ in women with previous caesarean section and $6.1 \%$ of all ectopic pregnancies [2, 3]. In 2012, Shen et al. [4], in their series of 45 patients with CS ectopic pregnancies, in which 42 women $(93.3 \%)$ had undergone only one caesarean section, concluded that multiple caesarean sections may not increase the risk of this condition. Similarly, systemic review found that scar ectopic pregnancy occurred in $52 \%$ of cases following prior one caesarean section, $36 \%$ in prior two caesarean section and $12 \%$ after three or more prior caesarean section [5]. It was diagnosed at the gestational age of 5-12 weeks with time interval between the last caesarean section and CS ectopic pregnancy was 6 months to 12 years [3].

\section{Pathophysiology}

Mechanism of this condition remains uncertain. It is possible that scar implantation occurs due to defects in the scar in the form of microtubular tract which develops due to poor healing of the previous trauma caused by caesarean section, dilatation and curettage, hysterotomy, myomectomy, abnormal placentation and manual removal of placenta [6]. Scar ectopic pregnancy is different from that of an intrauterine pregnancy with placenta accreta. In placenta accreta, the absence of decidua basalis leads to varying degrees of invasion of the myometrium by trophoblastic tissues, but pregnancy is primarily in uterine cavity. In scar ectopic pregnancy, gestational sac is completely surrounded by myometrium and fibrotic tissue of the scar and is separated from the endometrial cavity.

Two different types of scar ectopic pregnancies are identified. Type I is caused by implantation in the prior scar with progression towards the cervicoisthmic (in prior caesarean section) space or the uterine cavity. Type II is caused by deep implantation into scar defect with infiltrating growth into the uterine myometrium and to uterine serosal surface which may result into uterine rupture and massive haemorrhage in first trimester of pregnancy which is most dangerous.

\section{Presentation and Diagnosis}

The most frequent symptom is painless vaginal bleeding, and the mean gestational age is under $7.5+/-2.5$ weeks. Rotas et al. [5] reported in their series that $37 \%$ women were asymptotic, $39 \%$ women had scanty, painless vaginal bleeding, and $16 \%$ had mild-to-intense pain in abdomen.
Nine per cent of women had only pain in abdomen. In 2011, Yu et al. [7] reported in their series of 100 cases with scar ectopic pregnancies that $45 \%$ patients were asymptomatic, $55 \%$ had vaginal bleeding, and $7 \%$ had pain in lower abdomen.

Many times, it does not have any specific symptom and can be easily misdiagnosed. This can lead to life-threatening haemorrhage during pregnancy or curettage, uterine rupture, disseminated intravascular coagulation and even a death. Sometimes, undiagnosed scar ectopic pregnancy can present with heavy bleeding, haemoperitoneum and shock after termination of early pregnancy or missed abortion [8]. Hence, early and accurate diagnosis is important for effective treatment to prevent these catastrophic complications.

The ultrasound was the first widely used method in diagnosing scar ectopic pregnancy. It has been reported that with improved ultrasound imaging, correct early diagnosis of such pregnancies is possible with sensitivity of $84.6 \%$ [5]. Experience sonologists may give the accurate diagnosis which will help in timely effective management. Diagnosis depends on symptoms, clinical manifestation, history of previous scar, serum $\beta \mathrm{hCG}$ level trasvaginal sonography (TVS). TVS diagnosis includes (1) an empty uterine cavity and cervical canal, (2) an gestational sac located at the anterior wall of the isthmic portion, separated from endometrial cavity or fallopian tube in previous caesarean scar, (3) a gestational sac embedded within the myometrium and the fibrous tissue of caesarean section scar at the lower uterine segment with an absence of defect in the myometrium between the bladder and the sac and (4) a high-velocity low-impedance vascular flow surrounds the gestational sac [4, 9]. However, it is difficult to differentiate scar ectopic pregnancy from anterior cervical ectopic pregnancy, inevitable abortion or a cervicoisthmic pregnancy [10]. Hence, high-resolution and colour ultrasound scanning is essential for differential diagnosis.

In 2012, Shen et al. [4] reported that in their series of 45 patients with scar pregnancies 21 patients were initially misdiagnosed with spontaneous abortion or cervicoisthmic pregnancy. In cervical pregnancy, on ultrasonography (USG), uterus is empty with an endometrial stripe and the gestational sac is seen in cervix giving an hour glass shape to the uterus with ballooned cervical canal. In inevitable abortion, gestational sac is seen in cervical canal and on colour Doppler sac appears avascular with exponential fall in serum $\beta \mathrm{hCG}$ level. In cervicoisthmic pregnancy, a layer of healthy myometrium is visible between bladder and gestational sac on USG.

Diagnosis of scar ectopic pregnancy is relatively easy in early pregnancy. It is recommended that magnetic resonance imaging (MRI) can be performed when diagnosis by transvaginal colour Doppler USG is difficult. 


\section{Management}

The aim of the management is to prevent the massive haemorrhage and conserve the uterus for further fertility and health and quality of life of woman. So far, various interventions have been proposed, but there is no consensus on the optimal therapeutic protocol for scar ectopic pregnancy. The treatment approach depends on various factors like gestational age, haemodynamic stability, availability of endoscopic expertise, further fertility and feasibility of serial follow-up by serology and imaging.

Conservative medical management includes systemic methotrexate, local embryocides or combination of both. Surgical management to be offered to the patients who are haemodynamically unstable or in patients who have failed with medical therapies and includes hysteroscopy, laparoscopy, laparotomy and uterine artery embolization

Non-invasive therapy with systemic methotrexate may be considered when gestational age is not more than 8 weeks and without foetal cardiac activity. It had been reported that when serum $\beta$ hCG levels were less than $5000 \mathrm{mIU} / \mathrm{ml}$ and myometrium thickness was less than $2 \mathrm{~mm}$, then systemic methotrexate was successful. Systemic methotrexate had success rate of $71-80 \%$ with only $6 \%$ requiring hysterectomy.

In cases with the poor vascularization of fibrous scar, absorption of system methotrexate can be limited. In such cases, local administration of methotrexate is more effective. Seow et al. [3] in their series of 12 cases of caesarean section ectopic pregnancy during the period of 6 years concluded that TVS- or TAS-guided methotrexate injection emerged as treatment of choice to terminate CS ectopic pregnancy. Regression of scar ectopic mass occurred between 2 months and 1 year. However, methotrexate was reported to have high rate of failure with side effects which required further treatment or even emergency hysterectomy. Apart from methotrexate, local injection of potassium chloride, hyperosmolar glucose, etoposide and crystallize trichosanthin has been reported.

In 2007, Wang et al. [11] reported heterotopic caesarean scar pregnancy following IVF-embryos transfer where TVS-guided KCL was injected to terminate CS embryo followed by healthy neonate delivered 6 months later.

Various regimes with combined medical treatment have been described. Medical treatment can interrupt pregnancy, but bleeding can continue for longer time; hence, medical treatment combined with surgical aspiration of sac has been recommended in certain cases, e.g. systemic methotrexate with TVS-guided sac aspiration, TVS-/TASguided sac aspiration with systemic or local methotrexate, local potassium chloride with TVS-guided sac aspiration with local or systemic methotrexate. These regimes can avoid unnecessary laparotomy and preserve the women's fertility, but long-term follow-up with serum $\beta$ hCG and imaging is necessary. It may take $4-16$ weeks for serum $\beta h C G$ to drop to non-pregnant stage. On follow-up scan, if high peak systolic velocity on Doppler persists, then these women are at high risk of internal bleeding or uterine rupture and hence require surgical procedure.

In scar ectopic pregnancy, gestational sac is not in the uterine cavity and chorionic villi implants on scar. Hence, trophoblastic tissue is unreachable to curette. Repeated attempt can cause uterine perforation leading to severe haemorrhage. If there is profuse bleeding during curettage and absent of product of conception, must proceed immediately for laparoscopy/laparotomy. In a review of literature by Arslan et al. [12] reported that out of nine women, eight required surgical intervention following unsuccessful curettage and complications of procedure.

To prevent and control profuse bleeding, various haemostatic majors in form of intrauterine balloon tamponade by Foley's catheter, local injection of vasopressin, shirodkar's suture, prior selective uterine artery embolization and bilateral uterine artery ligation are advised.

Hysteroscopic evacuation of scar ectopic pregnancy requires general anaesthesia, operative skill and infrastructure. Direct visualization of scar pregnancy with careful evacuation and coagulation of blood vessels at the implantation site prevent massive haemorrhage. Chao et al. [13] reported successful hysteroscopy evacuation of scar pregnancy who had continuous vaginal bleeding and persistent serum $\beta$ hCG level following curettage and methotrexate.

Successful laparoscopic resection of scar pregnancy was first performed by Lee et al. [14] Principles of laparoscopic surgery are: scar pregnancy is excised and removed in endobag, bleeding is minimized by local injection of vasopressin, haemostasis is achieved by bipolar diathermy, and the uterine defect is closed by endoscopic suturing. Laparoscopic approach is more justifiable when scar pregnancy is deeply implanted and grows towards abdominal cavity and bladder. In 2013, Wang et al. [15] reported that 11 patients with scar pregnancy underwent successful laparoscopic bilateral uterine artery ligation and resection of the scar with gestational tissue and wound repair or laparoscopic bilateral uterine artery ligation combined with transvaginal resection of the scar with gestational tissue and wound repair with preservation of uterus.

Primary open surgical treatment should be considered in patients who do not respond to medical and/or other surgical treatments, when presented too late and in case of non-availability of endoscopic expertise and facilities. Exploratory laparotomy is necessary when uterine rupture is confirmed or strongly suspected. Some obstetricians recommend as best treatment option due to complete removal of scar pregnancy with repair of scar and quick return of serum $\beta \mathrm{hCG}$ level to normal in 1-2 weeks. 
Excision and repair of old scar result in removal of microtubular tracts and thus reduce the risk of recurrence.

In 2011, Rotas et al. [5] reviewed 112 cases and reported that expectant management of 6 patients resulted in uterine rupture and three of them required hysterectomy. Dilatation and curettage were associated with severe maternal morbidity. Out of 12, eleven patients had successful wedge resection with repair of implantation site through laparotomy or laparoscopy. Five women with $\beta \mathrm{hCG}$ exceeding $10,000 \mathrm{mIU} / \mathrm{ml}$ had successful outcome of systemic and local administration of methotrexate.

In clinical practice, bilateral uterine arterial chemoembolization has been tried out for scar ectopic pregnancy. Methotrexate is administrated directly into the gestational foci through bilateral uterine arteries followed by injection of occlusive agent which will block the feeding vessel. This causes chemotherapy as well as tissue ischaemia resulting in higher and higher concentration of methotrexate to the gestational foci; hence, more effective embryocide and less systemic toxic effects compared to embolization alone are observed.

In 2012, Shen et al. [4] reported that 45 patients were successfully treated with uterine artery embolization combined with local methotrexate. One patient had massive vaginal bleeding after 20 days of treatment which required emergency hysterectomy. The mean time for normalization of serum $\beta$ hCG was 37.7 days, and scar ectopic mass disappearance was 33.3 days.

It is high time to consider steps for prevention of scar ectopic pregnancy. Literature is conflicting regarding single-layer versus multi-layer uterine closure on the development of uterine scar defects. A change in uterine closure technique may improve the healing of uterine scar and consequently decrease in the rate of this condition.

After all modalities of conservative management of scar ectopic pregnancy, successful viable intrauterine pregnancies have been reported. Seow et al. [3] reported incidence of $50 \%$, with the range of 3-34 months between the previous CS ectopic pregnancy and subsequent pregnancy. One woman died of uterine rupture in the following pregnancy at $38+3$ weeks. Very few cases of recurrence of scar ectopic pregnancy have been reported.

\section{Conclusion}

Scar ectopic pregnancy is a dangerous and complex disorder with increasing occurrence in recent years. Accurate early diagnosis and effective management are important to reduce maternal mortality and mortality. Effective treatment of scar ectopic pregnancy should be carried out in first trimester to achieve the optimal treatment objectives, including termination of pregnancy before rupture, resection of pregnancy mass with proper suturing which will preserve future fertility and prevent recurrence.

\section{Compliance with ethical standards}

Conflict of interest None.

\section{References}

1. Larsen JV, Solomon MH. Pregnancy in a uterine scar sacculus: an unusual cause of postabortal haemorrhage. S Afr Med J. 1978;53:142-3.

2. Jurkovic D, Hillaby K, Woelfer B, et al. First-trimester diagnosis and management of pregnancies implanted into the lower uterine Caesarean section scar. Ultrasound Obstet Gynecol. 2003;21:220-7.

3. Seow KM, Huang LW, Lin YH, et al. Caesarean scar pregnancy: issues in management. Ultrasound Obstet Gynecol. 2004;23:247-53.

4. Shen L, Tan A, Zhu H, et al. Bilateral uterine artery chemoembolization with methotrexate for cesarean scar pregnancy. AJOG. 2012;207:386 e1-6.

5. Rotas MA, Haberman S, Levgur M. Cesarean scar ectopic pregnancies: etiology, diagnosis and management. Obstet Gynecol. 2006;107:1373-7.

6. Rosen T. Placenta accreta and cesarean scar pregnancy: overlooked costs of the rising cesarean section rate. Clin Perinatol. 2008;35(3):519-29.

7. Yu XL, Zhang N, Zuo WL, et al. Cesarean scar pregnancy: an analysis of 100 cases. Zhonghua Yi Xue Za Zhi. 2011;91(45):3186-9.

8. Timor-Tritsch IE, Monteagudo A, Santos R, et al. The diagnosis, treatment and follow-up of cesarean scar pregnancy. Am J Obstet Gynecol. 2012;207:44-6.

9. Pascual MA, Hereter L, Graupera B. Three-dimensional power Doppler ultrasound diagnosis and conservative treatment of ectopic pregnancy in a cesarean section scar. Fertil Steril. 2007;88:706.e5-7.

10. Ash A, Smith A, Maxwell D. Caesarean scar pregnancy. Int J Obstet Gynecol. 2007;114:253-63.

11. Wang CN, Chen CK, Wang HS, et al. Successful management of heterotopic cesarean scar pregnancy combined with intrauterine pregnancy after in vitro fertilization-embryo transfer. Fertil Steril. 2007;88(3):706.e13-6 (Epub 2007 Aug 6).

12. Arslan M, Pata O, Dilek TU, et al. Treatment of viable cesarean scar ectopic pregnancy with suction curettage. Int $\mathrm{J}$ Gynecol Obstet. 2005;89:163-6.

13. Chao A, Wang TH, et al. Hysteroscopic management of cesarean scar pregnancy after unsuccessful methotrexate treatment. J Minim Invasive Gynecol. 2005;12(4):374-6.

14. Lee CL, Wang CJ, Chao A, et al. Laparoscopic management of an ectopic pregnancy in a previous caesarean section scar. Hum Reprod. 1999;14:1234-6.

15. Wang HY, Zhang J, Li YN, et al. Laparoscopic management or laparoscopy combined with transvaginal management of type II cesarean scar pregnancy. JSLS. 2013;17(2):263-72. 